\title{
Keynes: ley de Say y demanda de dinero.
}

\author{
Brayan Alexander Baron Ortegon. \\ babarono@unal.edu.co
}

\section{Resumen}

En esta crítica bibliográfica, se hace un recuento de los principales argumentos de JM Keynes, contra la Ley de Say, considerada por él, el núcleo básico de la economía clásica y un caso especial, de una teoría mucho más amplia y general y por lo tanto mucho más potente en materia de política económica. Estos argumentos son esenciales, para entender la economía keynesiana y sus aportes posteriores, en teoría económica y política económica.

\begin{abstract}
In this bibliographic review, the Keynes'main arguments against the Say's law are gathered, this law was thought to be a special case of a broader and general theory and therefore more powerful as economic policy is concerned. These insights are vital, to understand Keynesian economics and its subsequent contributions in economic theory and economic policy.
\end{abstract}

\section{Palabras clave:}

Keynes, Ley de Say, Teoría económica, Demanda de dinero, Incertidumbre, Expectativas, Oferta agregada, Demanda agregada, Economía monetaria, Historia del pensamiento económico.

\section{Introducción}

"El núcleo de la crítica keynesiana puede sintetizarse como el rechazo puro y simple de lo que ha venido a llamarse la ley de Say.” Paul Sweezy (1946).

La economía clásica y neoclásica (De aquí en adelante clásica), desde los tiempos de Smith, Ricardo y Say, pasando por Walras y Marshall, tiene como uno de sus principios fundamentales la ley de Say. Keynes ponen en duda, la validez general de este principio y por lo tanto de sus corolarios en materia práctica y política económica. 
La ley de Say, plantea que la economía tiende hacia un equilibrio permanente entre oferta y demanda agregada y cualquier desajuste es solo temporal. Dichos desequilibrios, pueden darse por la intervención estatal, información incompleta, no racionalidad de los agentes, o cualquier otra distorsión, falla de mercado o de los supuestos del modelo clásico. Suponiendo una estructura de mercado de competencia perfecta, con el suficiente tiempo y sin la presencia de la distorsión, las fuerzas del mercado, guiadas por las señales de los precios reales, llevaran necesariamente a un equilibrio entre oferta y demanda agregada. El nuevo equilibrio, maximiza la eficiencia de los recursos disponibles, en función de las dotaciones iniciales de los agentes y sus preferencias, además es óptimo en el sentido de Pareto (Primer teorema de la economía del Bienestar). En 1936, Keynes vendría a cuestionar este postulado teórico, diciendo que los desajustes no necesariamente son temporales, sino que en realidad son inherentes y estructurales, al tipo de economía monetaria predominante en el siglo XIX y XX. Para Keynes, los supuestos de la teoría clásica son simplificaciones tan grandes de la realidad que solo son válidas en un caso especial.

Más aun, las características del caso especial supuesto por la teoría clásica no son las de la sociedad económica en que hoy vivimos, razón por la que sus enseñanzas engañan y son desastrosas si intentamos aplicarlas a los hechos reales. (Keynes, 1936, p. 15)

Por lo anterior, la crítica de Keynes, contra la ley clásica de Say, es una a su validez general y por lo tanto a su pertinencia a la hora de ejecutar política económica. La crítica de Keynes a la ley de Say, yace principalmente en dos elementos la eficiencia marginal del capital (EMK) y la demanda de dinero. El dinero es un bloque fundamental del edificio keynesiano, para Keynes, la economía es esencialmente monetaria, el dinero es más que un medio de intercambio, reserva de valor y unidad de medida, es un bien con demanda propia, usado por los agentes para formar sus expectativas sobre el futuro y cuya obtención es el objetivo principal de los individuos.

La validez general de la ley de Say es incompatible con la existencia del ciclo económico, en contraste para Keynes, el ciclo económico no solo es una cuestión de un desajuste temporal de la economía, es un fenómeno permanente, cuyas causas están siempre presentes: incertidumbre, patrón de comportamiento de los agentes y su relación con el dinero. 


\section{Ley de Say}

(...) Un producto terminado ofrece, desde ese preciso instante, un mercado a otros productos por todo el monto de su valor. En efecto, cuando un productor termina un producto, su mayor deseo es venderlo, para que el valor de dicho producto no permanezca improductivo en sus manos. Pero no está menos apresurado por deshacerse del dinero que le provee su venta, para que el valor del dinero tampoco quede improductivo. Ahora bien, no podemos deshacernos del dinero más que motivados por el deseo de comprar un producto cualquiera. Vemos entonces que el simple hecho de la formación de un producto abre, desde ese preciso instante, un mercado a otros productos. (Say, J.B, Tratado de Economía Política, 1841, p. 124)

La anterior cita es la definición de la ley de Say, hecha por el mismo Say. Básicamente dice que: los costos incurridos en la producción global o los pagados a todos los dueños de los factores de producción usados, son íntegramente gastados en la misma producción. Lo que implica que los empresarios, rentistas y obreros gastan el total de sus ingresos ya sea en consumo o en ahorro, destinando este último al mercado, para que se conviertan en inversión productiva. Es decir que, según esta ley, el simple hecho de producir genera la capacidad de demanda de la misma producción, o sea nunca puede haber un exceso general de producción, ya que siempre están garantizados los ingresos o la capacidad de compra de esta misma.

La ley de Say no niega la posibilidad de sobreproducción relativa en un sector de la economía o mercado, porque los productores podrían no disponer de toda la información relevante y no ser suficientemente previsores de las cantidades que se demandaran. Esta sobreproducción parcial afectaría los precios relativos de los bienes de los sectores que transan con el sector con exceso de oferta. Sin embargo, el exceso de oferta solo puede ser temporal, pues suponiendo libre competencia y precios relativos flexibles, estos últimos serán corregidos por las fuerzas del mercado.

Una versión primitiva de la ley de Say, ya estaba presente en algunos economistas, como Adam Smith (Rodríguez, 2003), por ejemplo, este en su obra capital dice:

La parte que anualmente se ahorra, se emplea inmediatamente como capital con el objeto de obtener beneficios, y se consume de la misma forma y aproximadamente en el mismo período de tiempo, pero por gente diversa, por trabajadores, manufactureros 
y artesanos que reproducen con un beneficio el valor de su consumo anual. (Smith, (1776), Citado por Rodríguez, (2003), p. 85)

Aunque implícitamente ya estaba en Smith, la ley debe su nombre al trabajo del economista francés Jean Baptiste Say, quien, con su Tratado de economía política, la popularizo. Siendo aceptada por Ricardo y John Stuart Mill (Rodríguez, 2003).

La escuela neoclásica, aunque con una concepción diferente a la escuela clásica en algunos aspectos como el método de análisis, la teoría del valor, el papel de la sociedad y la historia etc. comparte con la ultima la idea de autorregulación y equilibrio de la economía, aceptando la validez general de ley de Say. Por ejemplo, Alfred Marshall la definiría así:

El ingreso total de una persona se gasta en la compra de bienes y servicios, Cierto que generalmente se dice que un hombre gasta parte de su ingreso y ahorra la otra: pero es un axioma económico muy conocido que el hombre compra trabajo y bienes con aquella parte de su ingreso que ahorra, del mismo modo que lo hace con la que gasta. Se dice que gasta cuando procura obtener satisfacción presente de los bienes y servicios que compra, y que ahorra cuando el trabajo y los bienes que compra los dedica a la producción de riqueza de la cual espera derivar medios de satisfacción en el futuro. (Marshall, Pure Theory of Domestic Values, p. 34, citado por Keynes, 1936, p. 28)

La escuela neoclásica, pulió la ley de Say y la formalizo, incorporándola en su modelo más importante: la teoría de equilibrio general, a través de la famosa ley de Walras. Según Lange (1942), esta ley establece que para determinado nivel de precios (vector de precios) (Pi) de n bienes, la suma de los valores de las demandas individuales (di) de toda la economía, es igual a la suma de los valores de todas las ofertas de la economía (Si).

$$
\sum_{i=1}^{n} p i d i+\sum_{i=1}^{n} p i s i \equiv 0
$$

La ecuación (2.1) garantiza que la totalidad de los mercados se vacíen, si existiera un desequilibrio por un exceso de oferta en algún mercado o un exceso de demanda en algún otro, los precios relativos de estos mercados cambiarían y se equilibraría de nuevo la economía y por definición se cumpliría la ley de Say (Rodríguez, 2003).

Una vez aceptada la ley de Say, los corolarios que se pueden sacar, según Gaviola (2001) son: 
i. Entre mayor sea la parte de ingreso que no se consume o el ahorro, mayor será el nivel de inversión productiva, por lo tanto, mayor será el crecimiento de la producción y crecimiento de la economía.

ii. La función del dinero es ser medio de cambio, los individuos no lo demandan sino exclusivamente para realizar los intercambios, ya que, si lo demandaran, valga la redundancia, la demanda por los bienes y servicios producidos sería menor y la ley de Say no se cumpliría.

iii. No hay límite para el crecimiento de la economía, excepto el del pleno empleo, ya que todo aumento de la producción garantiza su propia demanda.

iv. Es imposible tener una sobreproducción agregada, ya que siempre existirá la forma de pagar la producción o lo que es lo mismo siempre estará asegurada la demanda agregada.

Para Keynes, las conclusiones de la escuela clásica y la neoclásica respecto a la ley de Say son básicamente las mismas, por eso es que incluye a la Escuela clásica: Ricardo, John Stuart Mill y sus seguidores (exceptuando a Malthus y Marx) y la escuela neoclásica en la categoría de economistas "clásicos" a estos es a los que Keynes dirige su crítica.

Desde los tiempos de Say y Ricardo los economistas clásicos han enseñado que la oferta crea su propia demanda - queriendo decir con esto de manera señalada, aunque no claramente definida, que el total de los costos de producción debe necesariamente gastarse por completo, directa o indirectamente, en comprar los productos. (Keynes, 1936, p. 28)

\section{Critica de Keynes a la ley de Say}

\subsection{Neutralidad del Dinero}

Ahora bien, sospecho que las condiciones necesarias para la 'neutralidad' del dinero, en el sentido que la suponen los PrincipIes of Economics de Marshall, para mencionar de 
nuevo este libro como un ejemplo representativo son precisamente las mismas que aseguran que no haya crisis (Keynes, 1973, p.1933).

Para Keynes la ley de Say se cumpliría si se cumple la condición clásica de neutralidad del dinero, Keynes la llama economía de trueque o real. En ella, el dinero es usado solamente para el intercambio, su función es puramente instrumental, es decir nadie demanda dinero por sí mismo, el dinero es neutral: no influye en las decisiones económicas que toman los individuos sobre los bienes y servicios que demandaran u ofertaran.

Bajo las condiciones de una economía de trueque, el dinero siempre permanecería en circulación y el consumo y el ahorro de todos los individuos se invertiría integralmente en la producción. Para Keynes, estas condiciones no se aplican a un tipo de economía monetaria, ya que en esta última el dinero se demanda no solamente como instrumento, para realizar intercambios, sino existen varios motivos, por los que existe una demanda de dinero inactivo que impide alcanzar una demanda efectiva igual o compatible con la plena capacidad de las fuerzas productivas de la sociedad y por ende alcanzar el pleno empleo. Las principales de estas razones, Keynes (1936, pp. 176-179), las cobijaría bajo el concepto de preferencia por liquidez y las definiría así:

1. Motivo transacción: el efectivo que las personas y empresarios necesitan para los intercambios normales.

"No obstante, y puesto que no hay forma de conservar poder general de compra salvo en dinero, la demanda de poder de compra se transforma en la de una demanda de una cantidad de dinero equivalente" (Keynes, 1930, p. 67)

2. Motivo precaución: como un "salvavidas" ante las eventualidades positivas y negativas que el futuro puede presentarle a los individuos.

3. Motivo negocios: los empresarios demandan cierta cantidad de dinero, para sobrevivir en el periodo de tiempo en que empieza la producción y reciben su ingreso.

4. Motivo gasto de consumo: las personas demandan cierta cantidad de dinero para sobrevivir en el periodo de tiempo, mientras les pagan sus ingresos.

5. Motivo especulación: es la demanda de dinero, si los individuos esperan una tasa de interés futura mayor que la actual. 
6. Conservación de riqueza: Según Keynes la única "justificación inteligente" para la existencia de la preferencia por liquidez, por el motivo 6 es: "Esta condición necesaria es la existencia de incertidumbre, respecto al futuro de la tasa de interés" (Keynes, 1936, pp. 151-152). Es decir, si es imposible predecir los rendimientos futuros, los individuos pueden comprar activos financieros o como los llama Keynes "Valores", pero siempre estarán en riesgo de incurrir en perdidas y es por esta razón que siempre habrá una demanda por liquidez de los individuos que no asumen el riesgo de comprar estos activos:

Por eso si puede concebirse que surja la necesidad de dinero efectivo liquido antes de que expiren los $\mathrm{n}$ años, existe el riesgo de incurrir en perdida al comprar una deuda a largo plazo y convertirla después en efectivo, en vez de haber conservado dinero en esta forma. (Keynes, 1936, p. 153)

Para Keynes una medida de la preferencia por liquidez es su costo de oportunidad o tasa de interés, que representa lo que las personas están dispuestas a renunciar por tener dinero en los bolsillos. Para determinado nivel de ingreso y determinada oferta monetaria, en realidad la tasa de interés no es lo que los economistas clásicos dicen: "el precio que equilibra la demanda y oferta de fondos prestables, en el mercado de ahorro-inversión” (Keynes, 1936, pp., 151-152)

En mi opinión, la principal razón para que no se haya resuelto el problema de las crisis, o para que la teoría disponible sea tan insatisfactoria, es la carencia de lo que podría denominarse una teoría monetaria de la producción. (Keynes, 1973, p.1)

\subsection{Dinero y Demanda de dinero}

El hecho de que parte del ingreso de cada individuo, no se consuma, ni tampoco se ahorre o invierta en el sistema financiero, se debe a que una parte de este ingreso se demanda en forma líquida o dinero, no solo para facilitar los intercambios, sino también debido a que la información y el contexto en el que están inmersos los agentes los conduce a esto, obstaculizando así que la demanda efectiva sea igual a la plena capacidad productiva de la economía; esto se debe a la existencia de incertidumbre, por eso los individuos buscan refugio, buscan protegerse ante las posibles eventualidades del futuro, que pueden ser positivas o negativas (Keynes, 1936).

Los individuos dependiendo de la coyuntura, demandaran una mayor o menor cantidad de dinero en el presente, si esperan que la tasa de interés sea más alta o más baja en el futuro, 
intentando así anticiparse a la incertidumbre y sobrellevar de una mejor manera los beneficios y riesgos que el futuro podría implicar:

"Esta condición necesaria es la existencia de incertidumbre, respecto al futuro de la tasa de interés" (Keynes, 1936, p.152)

Si no puede predecirse, cuál va a ser el costo de oportunidad de la liquidez, que es la tasa de interés, ni los rendimientos de este sacrificio, entonces los individuos con mayor aversión al riesgo demandarán una cantidad de dinero mayor en el presente, para proteger su riqueza de las fluctuaciones en la tasa de interés (Keynes, 1936).

Keynes (1936) afirma que los individuos demandaran más dinero ante la incertidumbre: ¿por qué un individuo que busca refugio ante la incertidumbre optaría por preservar su riqueza en forma de dinero, y no en forma de algún activo refugio, como, por ejemplo: el oro?, ¿acaso si hay incertidumbre sobre la tasa de interés, no es más probable que ante un golpe de la inflación o deflación, el valor del dinero varié más que el valor del oro? La respuesta más probable a este interrogante, nos la da Meltzer:

Keynes no tiene duda de cuál efecto domina. La incertidumbre incrementa la demanda por dinero y eleva la tasa de interés. Esta conclusión puede haber tenido un fundamento analítico, pero es más probable que estuviera basada en observaciones, particularmente sus observaciones de la inflación en Europa en los inicios de los años 20 (Meltzer, 1988, p.147, Traducción propia).

Keynes creía que los individuos se sienten más seguros, con dinero que, sin él, ya que el principal temor que genera la incertidumbre no es la pérdida del poder adquisitivo por una variación generalizada de los precios, pues los individuos los consideran, en general, estables, sino que el miedo en si es por la pérdida del ingreso mismo, por lo que demandan más dinero sin importar si es más o menos seguro que otros activos (Meltzer, 1988).

"Los poseedores de riqueza calman su inquietud acerca del futuro acumulando riqueza en forma de dinero, precisamente porque el mundo real es sumamente dinámico y el futuro es, ante todo, incierto." (Dudley, 1975, p.177).

En resumen, para Keynes (1936), la economía monetaria está caracterizada precisamente por esto, porque en ella el dinero, no es neutral para los individuos, sino que afecta la forma en que toman sus decisiones y la forma en que se comportan, ya que el dinero es útil para los individuos, en más de una forma, además de simplemente permitir el intercambio. 
Según Keynes (1936), Los clásicos cometen una imprecisión no lógica, pero sí de generalidad al analizar la economía monetaria, descartando las relaciones de tipo monetario diferentes al simple intercambio (unidad de medida, reserva de valor, medio de pago). Las cualidades que los clásicos suponían tenía la economía de "trueque" y bajo las cuales la ley de Say es válida en general, son diferentes de las cualidades de la economía en que vivimos o como la llama Keynes: la" economía monetaria", en esta última, las relaciones de tipo monetario son vitales, y afectan las decisiones y acciones de los individuos, es decir estas relaciones monetarias no son neutrales, haciendo que la producción no se demande totalmente, ya que una parte del ingreso se destina a la demanda de dinero, porque este le es útil a los individuos en más de una forma, además de instrumento del simple intercambio.

\subsection{Demanda efectiva}

El volumen de trabajo $\mathrm{N}$ que los empresarios deciden emplear depende de la suma (D) de dos cantidades, es decir, D1, la suma que se espera gastara la comunidad en consumo, y D2, la que se espera que dedicara a nuevas inversiones. D es lo que antes hemos llamado demanda efectiva. (Keynes, 1936, p. 36)

En una economía de tipo monetario, el ingreso no necesariamente se demanda totalmente, debido a que el dinero no es neutral, pero además no se demanda totalmente, a causa de que las demandas individuales están sujetas a una "ley psicológica fundamental", que hace que los individuos siempre ahorren un porcentaje de su ingreso, que está determinado por su propensión marginal a consumir (PMC), (Keynes, 1936). Si C es el consumo e Y el ingreso, el comportamiento de esta ley se puede expresar como:

$$
(\Delta \mathrm{Y} \rightarrow \Delta \mathrm{C} \wedge \Delta \mathrm{Y}>\Delta \mathrm{C}) \forall \Delta \mathrm{Y}
$$

La expresión (3.21) nos dice que todo incremento del ingreso $(\Delta \mathrm{Y})$ implica un incremento del consumo $(\Delta \mathrm{C})$, tal que este incremento del ingreso siempre es mayor que el incremento del consumo. La proporción en que aumenta el consumo ante un aumento del ingreso es del mismo signo que este y menor que la unidad (Keynes, 1936). 
Si $\mathrm{S}=$ Ahorro y $\Delta \mathrm{S}$ es un incremento del ahorro. Según Keynes (1936) el ahorro es siempre igual a la inversión (I).

$$
\begin{array}{r}
\mathrm{Y}-\mathrm{C} \equiv \mathrm{S} \vee \Delta \mathrm{Y}-\Delta \mathrm{C}=\equiv \Delta \mathrm{S} \\
\mathrm{S}=\mathrm{I}
\end{array}
$$

"Por consiguiente, en conjunto, el excedente del ingreso sobre el consumo, al que llamamos ahorro, no puede diferir de la adición al equipo de capital, al que llamamos inversión.” (Keynes, 1936, p. 64).

Por un momento, Keynes (1936) supone que no hay demanda por dinero y que los ahorros individuales a nivel agregado (S), se ofrecen en el mercado de fondos prestables, a determinada tasa de interés ( $\left.\mathrm{r}^{*}\right)$, que compensa el sacrificio de los individuos por su preferencia por liquidez, para determinado nivel de ingreso y oferta monetaria. Luego supone que los inversores demandan e invierten totalmente estos ahorros de los individuos, de modo que se equilibra el mercado ahorro-inversión o de fondos prestables, entonces $\left(\mathrm{r}^{*}\right)$ es la tasa natural de interés.

Por tanto, tipo de interés natural es el que hace iguales la inversión y el ahorro, de forma que el nivel general de todos los precios $(\pi)$ sea exactamente igual a la tasa monetaria de retribución de los factores de producción. (Keynes, 1930, p. 148).

Bajo las anteriores condiciones, la ley de Say se seguiría cumpliendo, ya que el faltante del ingreso que no se consume por los individuos, sería utilizado por los inversores, igualando así la oferta agregada y la demanda agregada. Pero, aunque la ecuación (3.23) es verdad siempre (en este punto Keynes concuerda con los "clásicos"), esto no necesariamente implica que siempre estén en equilibrio y que el total del ahorro se vuelva siempre inversión, ya que la dirección de causalidad es la opuesta y en realidad es la inversión la que determina el ahorro:

El análisis tradicional ha advertido que el ahorro depende del ingreso, pero ha descuidado el hecho de que este depende de la inversión, en tal forma que, cuando esta cambia, el ingreso debe cambiar necesariamente en el grado precisamente necesario para hacer que la variación en el ahorro sea igual a la de la inversión. (Keynes, 1936, p. $165)$. 
Bajo esta lógica keynesiana, una variación de la tasa de interés llevara a una variación del consumo y por lo tanto el ahorro, vía una variación del ingreso, pues este cambiara no en función del ahorro, sino en función de la variación en la inversión.

Como los ingresos bajaran en una suma absoluta mayor que la inversión, es indudable que, cuando la tasa de interés sube, la de consumo desciende; pero no quiere decir que por ello habrá un margen mayor para el ahorro; al contrario, ambos (ahorro y gastos) decrecerán. (Keynes, 1936, p. 105).

Pero según Keynes (1936), la demanda de inversión de los empresarios, que en última instancia determina el nivel de ahorro, de empleo y de producción depende de dos cosas:

La Eficiencia marginal del capital (EMK), que es la diferencia entre el "rendimiento probable de la inversión" y su precio de oferta.

La tasa de interés (r), lo que pagan los empresarios por pedir prestado el dinero que. No necesariamente es la natural.

La diferencia entre EMK y tasa de interés (r), determina el "aliciente" para invertir, entre mayor sea la magnitud de esta diferencia, mayor será el nivel de inversión de los empresarios, mayor será el nivel de empleo $(\mathrm{N})$ y mayor será la escala de producción. Entonces la demanda por inversión es función de:

$$
\text { Demanda de inversión = EMK- (r). }
$$

La EMK depende del rendimiento probable que son las expectativas sobre la rentabilidad futura de un bien de capital, estas dependen de las "creencias" de los empresarios sobre cuál va a ser el nivel de la demanda efectiva en el futuro, el nivel de tecnología y la rentabilidad futura de sus inversiones, del nivel de salarios etc. Comparadas con el costo de reposición del bien (Keynes., 1936). Estas creencias o previsiones están basadas en los rendimientos que les parezcan a los empresarios más probables y a la confianza de que esas previsiones van a cumplirse (Keynes, 1936).

La propensión a invertir depende de la renta que el empresario espera conseguir de la inversión corriente respecto del tipo de interés que tiene que pagar para financiar su producción o, para decirlo de otra manera, el valor de los bienes de capital depende del tipo de interés al que se capitalizan las rentas futuras derivadas de esa inversión. (Keynes, 1930, p. 147) 
Es decir, dependiendo de las expectativas empresariales, se demandará más o menos inversión, ello a su vez que determina el nivel de producción y por lo tanto de empleo (Keynes, 1936). Para Keynes (1936), no hay forma de que los empresarios puedan predecir estadísticamente (o siquiera de otro modo fiable) sus "esperanzas" o expectativas de la rentabilidad futura que es lo que determina la inversión presente y por lo tanto el nivel de inversión, empleo, producción y consumo. Esto implica que en una economía monetaria la capacidad productiva puede estar por debajo de su nivel máximo o puede haber desempleo involuntario, es decir no existen mecanismos que aseguren una inversión suficiente que lleve a una demanda efectiva en la economía compatible con la ocupación plena.

Por otro lado, la predicción es algo difícil y que requiere mucha más información de la disponible, de manera que los empresarios, por regla general, se dejan guiar por lo que les dice su experiencia, complementada con alguna clase de ideas muy generales acerca de las posibles consecuencias de los cambios en el tipo de interés bancario, la oferta de crédito y la situación de los intercambios con el extranjero. (Keynes, 1930, p. 151)

\subsection{Dinero y EMK}

“En mi opinión, la principal razón para que no se haya resuelto el problema de las crisis, o para que la teoría disponible sea tan insatisfactoria, es la carencia de lo que podría denominarse una teoría monetaria de la producción.” (Keynes, 1973, p.1)

La oferta de dinero junto a la preferencia por la liquidez determina la tasa de interés monetaria, que, dependiendo de las expectativas de los empresarios sobre el valor de la demanda futura, llevara a un nivel de inversión que puede ser o no compatible con el pleno empleo de los recursos productivos de la economía.

Estas expectativas, dependerán de la EMK: “La relación entre el rendimiento probable de un bien de capital y su precio de oferta o de reposición, es decir, la que hay entre el rendimiento probable de una unidad más de esa clase de capital y el costo de producirla, nos da la eficiencia marginal del capital de esa clase." (Keynes, 1936, p.125).

La EMK, ceteris paribus, dependerá en parte del rendimiento probable, que a su vez dependerá de las expectativas sobre el valor futuro del dinero, entendiendo este valor futuro por el monto que los empresarios creen que van a pagar en el futuro, para cubrir los costos de producción, de uso del capital, de almacenamiento, salarios, etc. Si las expectativas de los empresarios los 
llevan a pensar que el valor del dinero será menor, la inversión aumentará y con esta la producción y el empleo, ya que la EMK aumentará por un aumento del rendimiento probable esperado por los empresarios. Tendremos la situación contraria, si la expectativa del valor del dinero se incrementa, ya que así tendríamos una disminución del rendimiento probable del bien de capital y una disminución de la inversión (Keynes, 1936).

Si aceptamos, las anteriores premisas de Keynes (1936) como verdaderas, la lógica nos lleva a que el nivel de inversión y por lo tanto de producción y ocupación en una economía no depende, per se, de la EMK promedio, sino del valor absoluto de la diferencia entre esta y la tasa de interés monetaria; pues no tendría sentido económico (racionalidad, así sea limitada), para un empresario tener un alto volumen de inversión en un periodo, esperando que en los siguientes su rendimiento probable, sea alto, si a la vez espera que deba retribuir al dador de crédito, que permitió la mayor inversión, también un monto de dinero proporcionalmente alto. El caso, donde la tasa de interés sea alta, tampoco haría rentable para el empresario invertir, dada su preferencia por la liquidez, anulándose de esta manera el beneficio potencial monetario:

"La propensión a invertir depende de la renta que el empresario espera conseguir de la inversión corriente respecto del tipo de interés que tiene que pagar para financiar su producción o, para decirlo de otra manera, el valor de los bienes de capital depende del tipo de interés al que se capitalizan las rentas futuras derivadas de esa inversión." (Keynes, 1930, p. 147)

Si hay un incremento en la expectativa de la tasa de interés, ceteris paribus, debe haber un incremento en la inversión en el periodo actual, pues los costos de producción ahora serán menores, que los esperados en el futuro, permitiendo un rendimiento efectivo mayor en el periodo actual, respecto al que se espera habrá en el futuro: (Keynes, 1936):

"Es importante entender la dependencia que hay entre la eficiencia marginal de un volumen determinado de capital y los cambios en la expectativa, porque es principalmente esta dependencia la que hace a la eficiencia marginal del capital quedar sujeta a ciertas fluctuaciones violentas que son la explicación del ciclo económico.” (Keynes, 1936, p.132).

Para Keynes (1936), es precisamente la importancia de la EMK y su dependencia explicita de las estimaciones monetarias del futuro del valor de los bienes durables y de la tasa de interés, lo que es el puente principal, entre el presente y el futuro, lo que determina que el dinero se convierta en bienes durables, Plumbum aurum fit. Es esta conexión entre el pasado y el futuro, la clave para entender la dinámica en la economía monetaria. 


\subsection{EMK y ciclo económico}

La explicación de Keynes (1936) del ciclo económico depende de los determinantes de la inversión, pues de esta dependerá el nivel de empleo y de producción. Estos determinantes son principalmente la EMK y la tasa de interés:

El dinero es el puente temporal principal en la economía, entre el pasado, el presente y el futuro, pero no es el único, ya que para Keynes (1936), existe otra característica de todos los bienes, cuya misma denominación indica esta característica temporal, esta es la durabilidad de los bienes, que influye en la escasez relativa de estos, afectando los inventarios y por lo tanto influyendo sobre la EMK, esto se da a través de las expectativas, ya que la propiedad misma de un bien durable afecta el nivel de inversión del bien a través de las expectativas de los inversionistas, simplemente porque la vida útil del bien, se extiende en promedio más que la de otros bienes, (Keynes, 1936).

Siguiendo la lógica de Keynes (1936), la incertidumbre de la economía, la incapacidad de los individuos de predecir los rendimientos y la tasa de interés futura, sumado a una alta susceptibilidad de los agentes a las creencias desproporcionadas y a las expectativas extremas de lo que piensa la mayoría; lleva a que se cierna la crisis, transmitiendo así la falibilidad humana: individual, grupal y del mercado, al agregado económico; ya que Errare humanum est.

Para Keynes (1936), cuando lo único que sostiene la inversión es una EMK alta, debida no a factores físicos (abundancia de bienes de capital y su durabilidad) ni a factores monetarios (tasa de interés, preferencia y por la liquidez), sino a expectativas injustificadas sobre los rendimientos probables de la inversión; además si esto se da en un contexto económico, donde prevalece la incertidumbre, se refuerza la falibilidad humana y la del mercado, siendo así inevitable el advenimiento de la crisis y la constante presencia del ciclo económico. La estructura de la economía monetaria y las condiciones que le impone a los individuos, en especial la incertidumbre sobre los rendimientos de la inversión y sobre el futuro de la tasa de interés, etc. lleva en si misma el germen de la crisis y de la subutilización de los recursos productivos de la sociedad. 


\subsection{Condiciones de Recuperación de la crisis}

El papel único de los instrumentos monetarios, en especial la tasa de interés, en la recuperación es descartado por Keynes $(1930,1936)$, ya que este siempre le da más preponderancia en la explicación del ciclo a la caída de EMK, que, a una subida imprevista de la tasa de interés monetaria, debido a que, para él, la incertidumbre y la caída en las expectativas del rendimiento probable afectará primero y siempre a la EMK y luego este efecto se verá reforzado por un cambio en la tasa de interés. (Keynes, 1930).

Bajo la lógica de Keynes (1936). La recuperación solo se dará cuando se haya reparado la confianza por parte de los inversionistas, cuando estos puedan realizar nuevas inversiones, esperando un rendimiento probable lo suficientemente alto, como para incentivar de nuevo la ocupación y el ingreso; cuando los bienes de capital sean de nuevo lo suficientemente escasos, para hacerles creer a los individuos que pueden realizar nuevas inversiones y que estas no serán echadas en saco roto, cuando se pueda invertir porque los individuos piensan que pueden hacerlo, Possunt quia posse videntur (Keynes, 1936).

\section{Conclusión}

En la concepción de economía monetaria de Keynes (1936), no existe un mecanismo de autoajuste inmediato, que lleve al pleno uso de la capacidad productiva (pleno empleo), pues las condiciones para que esto suceda no son la del caso general, aunque tampoco se puede descartar lógicamente la posibilidad, debido a que el nivel de producción depende de la demanda efectiva, las expectativas y la tasa de interés, y solo en ciertas combinaciones de estas variables se da el caso especial de pleno uso de la capacidad productiva.

Para Keynes, en la economía monetaria la relación de causalidad entre oferta agregada y demanda agregada, va en sentido contrario al caso de la economía de trueque, donde se cumple la ley de Say, ya que en la economía monetaria las decisiones de demanda agregada son las que determinan la oferta agregada, a través de: 1) la (PMC) y un consumo menor al ingreso, 2) la demanda de inversión por las expectativas de los empresarios sobre sus ganancias futuras y su diferencia con la tasa de interés 3) la demanda de dinero por la preferencia por liquidez.

Por las anteriores razones la oferta y la demanda no son iguales, porque cada uno de estos, tiene en realidad causas diferentes. 
No existe la identidad de las funciones de oferta y demanda porque son diferentes. La curva de oferta tiene pendiente igual a uno y la demanda tiene una pendiente menor que uno por la propensión marginal al consumo, haciendo que la demanda se constituya en un obstáculo para el incremento de la producción. Keynes plantea el Principio de la Demanda Efectiva con el que desmiente la ley de Say. (Ana Cristina Argoti, 2003, p.56)

Para Keynes, las situaciones futuras son de incertidumbre, las posibles peripecias del mañana no son susceptibles de ser probabilizadas y no existen para ello métodos diferentes a la “convención" y experiencia. Por eso es que existe una demanda de inversión determinada por la EMK y la tasa de interés, por eso es que existe una demanda de dinero por especulación y por precaución, porque el dinero es como un salvavidas ante los posibles naufragios del futuro.

La ley de Say es un caso especial, ya que requiere supuestos muy fuertes: neutralidad del dinero, $(\mathrm{PMC})=1$, inexistencia de la preferencia por liquidez, determinación del nivel de inversión solamente en el mercado de fondos prestables etc. Estas condiciones no predominan en una economía monetaria, por lo tanto, la ley de Say no tiene validez general y sus corolarios tampoco, aunque no se puede descartar lógicamente su cumplimiento y dado que esta Ley es una condición necesaria (y bajo ciertas condiciones suficiente) para alcanzar el equilibrio general y que se puede demostrar lógicamente la existencia de este (Arrow y Debreu, 1954), aunque no se puede demostrar lógicamente su unicidad (Sonnenschein, 1972). Por lo anterior, la crítica de Keynes, no es una a la lógica ni al método de los clásicos, es una a la poca factibilidad y generalidad de sus premisas y por lo tanto a su poca potencia y efectividad en materia practica y de política económica.

\section{Referencias}

Argoti, Ana (2011). Algunos elementos sobre la teoría clásica del empleo y la versión Keynesiana. Revista de la Facultad de Ciencias Económicas y Administrativas. Universidad de NariñoVol. XII. No. 22do. Semestre 2011, (pp. 35-57). Recuperado de https://s3.amazonaws.com/academia.edu.documents/40617962/Dialnet-

AlgunosElementosSobreLaTeoriaClasicaDelEmpleoYLaVe3854586.pdf?AWSAccessKeyId=AKIAIWOWYYGZ2 Y53UL3 A\&Expires=1522275592\&Si

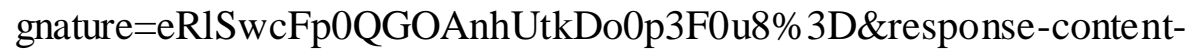
disposition=inline $\% 3 \mathrm{~B} \% 20$ filename\%3DAlgunos_Elementos_Sobre_La_Teoria_Clasic.pdf Arrow, K. J.; Debreu, G. (1954). "Existence of an equilibrium for a competitive economy". Econometrica. 22 (3): 265-290. doi:10.2307/1907353. 
Dudley. Dillard. (1975). La Teoría económica de John Maynard Keynes: teoría de una economía monetaria. Madrid: Aguilar s.a. ediciones.

Gaviola, Saúl. (2001). La ley de Say y la imposibilidad de la crisis según los teóricos clásicos. Recuperado de: http://www.academia.edu/426853/La_ley_de_Say_y_la_imposibilidad_de_la_crisis_seg\%C3 \%BAn_los_te\%C3\%B3ricos_cl\%C3\%A1sicos

Keynes, J.M. (1930). Tratado del dinero: teoría pura y aplicada del dinero. Madrid: Ediciones Aosta (Eds.) 1996. traducido por José Antonio de Aguirre. Madrid, España

Keynes, J.M. (1936). Teoría general de la ocupación, el interés y el dinero. México: Fondo de Cultura Económica.

Keynes, J.M. (1973). Una Teoría monetaria de la producción. Tomado de Keynes, J.M., 1973, "Towards the general Theory", The Collected Writings 01 JMK, v. XIII, St. Martin's Press, N. Y. Maya y Alberto Supelano (Trads).

Lange, Oskar. (1942). La ley de Say: reformulación y crítica, (pp. 23-42), Biblioteca del pensamiento económico, Banco central de Venezuela. Recuperado de http://www.bcv.org.ve/Upload/Publicaciones/rbcvs012.pdf

Meltzer, AH. (1988). Keynes's monetary theory: a different interpretation. Cambridge: University Press.

Rodríguez, J.C (2003). La economía laboral en el período clásico de la historia del pensamiento económico. Recuperado de http://uvadoc.uva.es/bitstream/10324/52/1/TESIS06-090318.pdf

Sardoni, Claudio. (1991). Marx y Keynes: la Crítica a la ley de Say. Tomado de Critica a la economía clásica, (pp. 183-201), Universitat Autónoma de Barcelona, (Eds.) Barcelona, España.

Say, J.B. (1841). Tratado de Economía Política, De los Mercados (pp. 121-129), México (DF), México: Fondo de Cultura Económica.

Sonnenschein, H. (1972). Market Excess Demand Functions. Econometrica, 40(3), 549-563. doi:10.2307/1913184Sweezy, Paul. (1946). Critica de la economía clásica. Ariel S.A (eds.). La aportación de Keynes al análisis del Capitalismo (pp. 97-108), Madrid, España. 